\title{
Smart Home Automation and Water Plantation Farming Using IOT
}

\author{
Prof. Chethan Raj C ${ }^{1} \mid$ Meghana $^{2}$ | Nischitha ${ }^{2}$ \\ ${ }^{1}$ Research Scholar \& Associate Professor, Computer Science and Engineering, UoM, Mysore, Karnataka, India \\ 2Student, Department of Computer Science and Engineering, Karnataka, India
}

To Cite this Article

Prof. Chethan Raj C, Meghana and Nischitha, "Smart Home Automation and Water Plantation Farming Using IOT", International Journal for Modern Trends in Science and Technology, 6(8S): 95-101, 2020.

Article Info

Received on 16-July-2020, Revised on 15-August-2020, Accepted on 25-August-2020, Published on 28-August-2020.

\section{ABSTRACT}

The project discussed in this paper is targeted at solving problems faced bypeople in their daily life. It is designed to control and monitor appliances via smartphone using Wi-Fi as communication protocol and raspberry pi as private server. All the appliances and sensors are connected to the internet via NodeMcu microcontroller, which serves as the gateway to the internet. Even if the user goes offline, the system is designed to switch to automated state controlling the appliances automatically as per the sensors readings. Also, the data are logged on to the server for future data mining. The core system of this project is adopted from the Blynk framework. This paper presents a low cost and flexible home control and environmental monitoring system. It employs an embedded micro - web server in NODE MCU microcontroller, with IP connectivity for accessing and controlling devices and appliances remotely. These devices can be controlled through a web application or via Bluetooth Android based Smart phone app. The proposed system does not require a dedicated server PC with respect to similar systems and offers a novel communication protocol to monitor and control the home environment with more than just the switching functionality. To demonstrate the feasibility and effectiveness of this system, devices such as light switches, power plug, temperature sensor, gas sensor and motion sensors have been integrated with the proposed home control system. Therefore this system has been successfully designed and implemented in real time. Using this we will be able to control home appliances through a web browser using your PC or mobile. These AC mains appliances will be connected to relays which are controlled by the NodeMCU ESP8266 and NodeMCU acts as a Web Server and we will send control commands through a Web Browser like Google Chrome etc. ESP8266 is the one of the most popular and low-cost Wi-Fi module available in the market today. The goal of this project is to develop a home automation system that gives the user complete control over all remotely controllable aspects of the home followed by smart farming or Dynamic planting system.

KEYWORDS: Internet of Things, Blynk app, NODEMCU, ESP8266, Temperature Detector, Soil Wetness Detector, Humidity Detector..

\section{INTRODUCTION}

In the running 21 century where social media is considered to be the most important activity, that helps people to gain more experience. We can simply say social networking through various media has changed the way we live. Due to the regular increase in internet users from all over the world social media has a substantial impact on 
humans, their communication, work and relationship in today's society, which has further increased the rate from millions to billions in terms of internet usage for operating social media. Social media has made people possible to communicate with anyone from anywhere at any time offering them to engage in social interactions [17].

In order to look after human behavior and manage such huge amount of data, Now-a-days social media is undergoing a lot of transformations and all thanks to technologies like machine learning or deep learning algorithms which helps researchers understand big data [1]. Machine learning hold big data sets in order to get insights and optimize social media. In fact, social media produce vey huge amount of data on daily basis. And to gain information about each individual user we need to translate that data. Machine learning or deep learning have helped researches to predict various human behavior patterns along with big data which makes it easy to store, retrieve and extract abundant information on humans and the data of their social activities can be stored and obtained in this big data era. The advancement in machine learning and big data have been helping researches to make predictions on wide range of future applications by analyzing various forms of patterns, considering this social technology have created an uprising in user-generated information, online human networking, and rich human behavior-related data. however, we can't tend to ignore the misuse of these social media pose a new form of serious threat, aggression, and violence against humans in the same online platform which is termed as cyberbullying. There are various studies to demonstrate that online platforms like social media are being used for bullying. And researchers have come up with various measures to stop cyberbullying and all these measures are manual process which takes a lot to time to process and detect threats. In order to solve this problem machine learning algorithm with big data are required to process this information quickly and introduce algorithms that can automatically detect threats [2].

Well the goal of this paper is to develop machine learning algorithms that can improve the manual monitoring of cyber bullying on online platforms into automatically detecting the aggressive human behavior from user end itself before they make a move to post threats. While a lot of research related to this focuses on detecting cyberbullying the present study focuses on a broad range of predicting cyber bullying by giving a form of filters with text- classification approach and lexicon based approach.

We propose a machine learning method to cyberbullying prediction by making use of random forest algorithm along with varied features. To our knowledge this is the first approach of using filter based word classification related to cyber bullying and in prediction of events like threats. For now, this paper focuses on single language i.e. filters based on only English well the methodology adopted can be used for varied other languages also.

\section{RELATED WORK}

K. Lova Raju [1] et.al presents that, the cost of the products is increasing in our routine life due to the development of technology a small idea called smart home project is introduced to reduce the cost and inconvenience. A smart home is able to control the home even though the person is not available in home. The IoT system can be formed by the collaboration of MCU with othercomponents like PIR sensor and ultrasonic sensor which are used to identify the movement of the any person while entering into the room. It gives notifications in mobile app.Data is uploaded into cloud using ThingSpeak so that data can be stored and retrieved whenever it is necessary.

Real Time clock based home automation in an advance project to control the devices in timely and systematic manner.RTC with EEPROM can record all the working parameters in the devices or appliances. The inbuilt RTC and EEPROM present in the controller will be activated for the operation. The controller also interfaced with WIFI to receive the control commands from Wi-Fi shield (Wi-Fi hotspot).As and when the request is received the microcontroller activates the RTC and EEPROM and as per request received the operation will be done. Once all the connections are given to all the components start working. This proposed system detects the person by the operation of different sensors. It gives notifications and alerts the user in different instances like when the person enters into the room and automatically switches on and off home appliances like fans, lights etc. For instance, if there is absence of light then immediately the bulb will glow.

Adel Gastli [2] et.al implemented the design of the Solar House monitoring system that is used for controlling the house energy efficient while maintaining the minimum required comfort-of-living conditions is implemented. While the sensors placed inside and around the house 
continuously Ventilation for Humidity, Temperature, and Air quality sensors. LDR Opening Case with a cover shaped like a tree leaf collect data, the information should be accessible at all times and uploaded every few minutes to the cloud server for display, processing, and archiving.The proposed design of the smart solar home is very flexible and can be easily expanded and applied to larger buildings by increasing the number of sensors, measured parameters, and control devices. More functionality and smartness could be also added to the existing system for making the house automation system grow, adapt, and evolve by itself using advanced artificial intelligence.

Solar House monitoring can help make you more aware of PV's system performance. It offers information about energy consumption, optimizing energy usage. The main objective of this technique is better energy utilization. The automation designed not just screens the sensor information, but also actuates different parameters as indicated by the necessity.

Satyendra K. Vishwakarma[3] et.al is implemented the working of the smart home automation as shown, initial requirement is the Internet connectivity to access their smart home appliance using an IFTTT statement command. It will be accessed through the Adafruit for creating the connectivity between the Google assistant and the NodeMcu which is the main control unit of the smart home automation. For continuous operation of the circuit, power backup is also provided with the help of rechargeable battery. Finally, with the help of Google assistant, based on the user command the home appliance can be turned $\mathrm{ON} / \mathrm{OFF}$ with the help of the designed system. In this paper, smart home automation controller unit and with the help of the design control unit, home appliance can be converted into a smart and intelligent device using IoT. Using the IoT connectivity, we can monitor and access our smart home easily from anywhere, which will definitely will prove to be energy efficient.Proposed system has two advantages. First, using the IoT connectivity, we can monitor and access our smart home easily from anywhere, which will definitely will prove to be energy efficient. Secondly, it act has a helping hand for the old age and differently abled person. For future work we would like to add up more controlling units that can make our smart home more intelligent that can be practically deployed in the real time situation.
Jayant Dorve [4] et.al refers to home automation via Android system. It links the connectivity with Wi-Fi router via Internet. In this the user will have to communicate with Raspberry Pi through the Internet via Wi-Fi network. The serial data coming from Wi-Fi is connected to Raspberry Pi circuit. The Raspberry Pi board is the main part of home automation. It has solid state relay(SSR) which is an electronic switching device which switches ON or OFF when external voltage is applied. The Wi-Fi unit is configured for communication and security. It has used keyboard and mouse for operation.

This project is divided into two sections. One is server side another is client side or user side. The server side is installed on Raspberry Pi whereas the client side is installed on mobile device. Server side is created with the help of LAMP (Linux, Apache, My SQL, PHP). It has used 40 GPIO pins of Raspberry $\mathrm{Pi}$ through the relay circuitry for operation. All appliances are connected to relay. User has to use mobile device to access Raspberry Pi via internet. IP address needs to be connect with Raspberry Pi with browser. The UI simply shows the number of rooms and present home appliances. Its programming is done in PHP language. The another advantage of this system is to help handicapped and old age people.

Akash Reddy [5] et.al describes that, the urban areas such as developed cities, the citizens have all the basic amenities like Electricity supply with minimal number of power cuts, Food supply, comparatively proper roads and infrastructure of buildings, etc. But same is not the case with rural areas, where majority of villages suffer electricity shortage, agricultural issues, improper supply and distribution of water for various purposes, etc. Hence through our project, Now, there are numerous factors that can influence the formation of a smart village. Crops are the watered using either ground water or irrigation from Hydraulic dams or Rain water. Depending upon the geographic location in the world, type of climate and type of soil, farmers select the type of crop for the season for maximum profit using maximum yield techniques. The irrigation system that we have designed and the rainfall prediction algorithm that we have implemented can be upgraded to higher and advanced levels. In this project, we aimed to propose a method for an effective irrigation system and an appropriate rainfall prediction algorithm. These two aspects if executed will accuracy, can prove as one of the pivotal factors that smartly utilizes all the resources provided to it. 


\section{PROBLEM STATEMENT}

Now days all have busy life for e.g. person staying somewhere for job and another someone comes and if user having an important meeting can't pick them and they don't have keys of home. This will put user in trouble. So with help of this project user locating in any place can control the device efficiently and effectively. If user working whole day in office and after your office work you don't want to do household works then home will do its own works like task of filling water, charging devices in absence of user/ home owner. If user come to home and your freeze will tell you what you can make in eating without opening it from the contents of food which are present in is then how awesome. If our daily life will become easily and less complex it will be than the golden era of technology. The problem statements leads to implement effective system to solve the problem by using application.

\section{SCOPE \& MOTIVATION}

The above said problem statement and related work leads to a motivation to implement an application. Day by day, the field of automation is blooming and these systems are having great impact on human beings. The project which is to be implemented is a home automation \& planting system using Easy IOT Webserver and WIFI and has very good future development. In the current system webserver is installed on a windows PC so the home appliances can be controlled using only by using the device on which webserver is installed. This can be further developed installing web server on cloud. Advantage of installing webserver on the cloud is that home can be controlled by using any device which has WIFI 802.1 and a web browser. By visiting the IP address of the cloud the control actions can be taken. Most smart home products are built with the intention of taking menial tasks out of people's lives. By getting rid of monotonous, daily activities, people will have more time to focus on other important activities.

The above problem statement and existing system leads a motivation to implements the smart home automation. With the advancement of technology, the need for efficient controlling is more as it optimizes performance and saves unnecessary wastage of power. The basic home appliances are fan, light and water pump which consume maximum power. Unnecessary wastage of power and resources by turning on lights during day time or high speed fans in winter season or water pump during overflow of water from tank can be avoided in this way. A system has been proposed to control home appliances anytime from anywhere in the world and efficiently utilize power by controlling appliances properly.

Its era of technology world is becoming faster and easier due to this reason our homes also should become part of it. Setting in any part of world where user can operate our home just by our smart phone with the help of node MCU and Blynk app. Here we use the concept called IOT. Using the concept of internet of things, the design of application is to make human life reliable. With the help of IOT things can be too easy. Main aim of this project is to atomize smart home and farming in less cost.

\section{PROJECT OBJECTIVE}

The project aims at designing an advanced home automation and planting system using normal web server and Wi-Fi technology. The devices can be switched ON/OFF and sensors can be read using a Personal Computer (PC) through Wi-Fi.

1.The project aims at designing an advanced home automation system using Node MCU ESP8266 wifi module.

2. The proposed system develops a home automation system that gives the user complete control over all remotely controllable aspects of the system.

3. The objective is to presents a low cost and flexible home control and environmental monitoring system.

4. The objective of system is to control home appliances anytime from anywhere in the world and efficiently utilize power by controlling appliances properly.

5. The system provides the implementation to be available in very less cost to ruler area peoples and schools.

\section{PROPOSED SYSTEM}

1. The proposed system introduces the advance era for home management system and security to make human life more easy and efficient and this system is applicable in real-time home automation, monitoring and controlling of remote systems efficiently.

2. This paper implements the problem faced by peoples in day to day life, main moto of this paper is to make things less complex and available in low prices.

3. It is mainly focused on overcoming everyday problems faced by the people in different places with lack of manpower in smart home, agriculture and farming, etc are blatantly evident. 
4. The proposed system uses the Node MCU and the IoT platform, and hence the application is cost effective and also uses Blynk app for user interface and to show the status of device that has been used to read data from sensors located in home environment and user controls home appliances based on these data.

5. This proposed work is made to help the farmers and make their harvest economical without the wastage of water and the consumption of power by motor can also be reduced so that they are conserved for the future use.

\section{SYSTEM ARCHITECTURE}

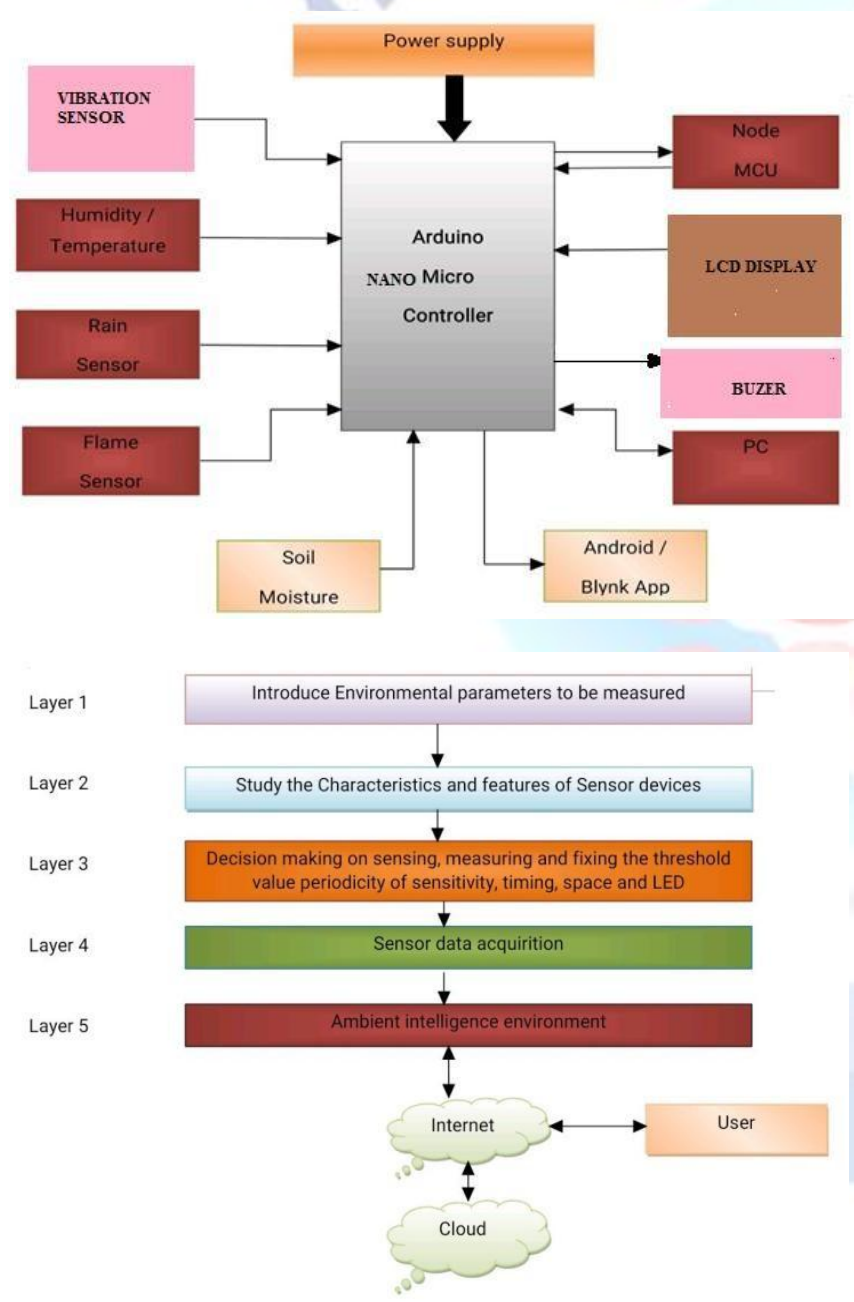

The results of the project $\&$ the snapshots for each of the activities are shown along with the discussion of each activity describing its working. Each snapshot describes every single step of the Application. The activities as well as the options provided to the users in each activity such as device status, Blynk app and all integrated devices are also shown and described in brief.

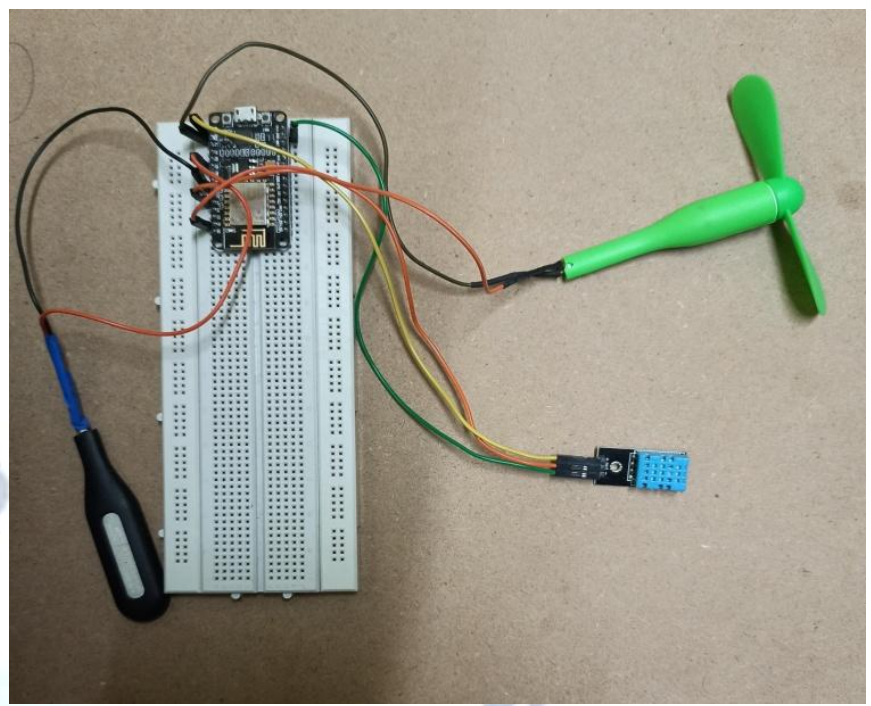

Fig 6.1: Smart Home Automation

The above diagram depicts the smart home automation that consists of node MCU, DHT sensor, LED and FAN. Based on the temperature the FAN switch to On or Off and also user can control or view the device status using web app or Mobile Devices.

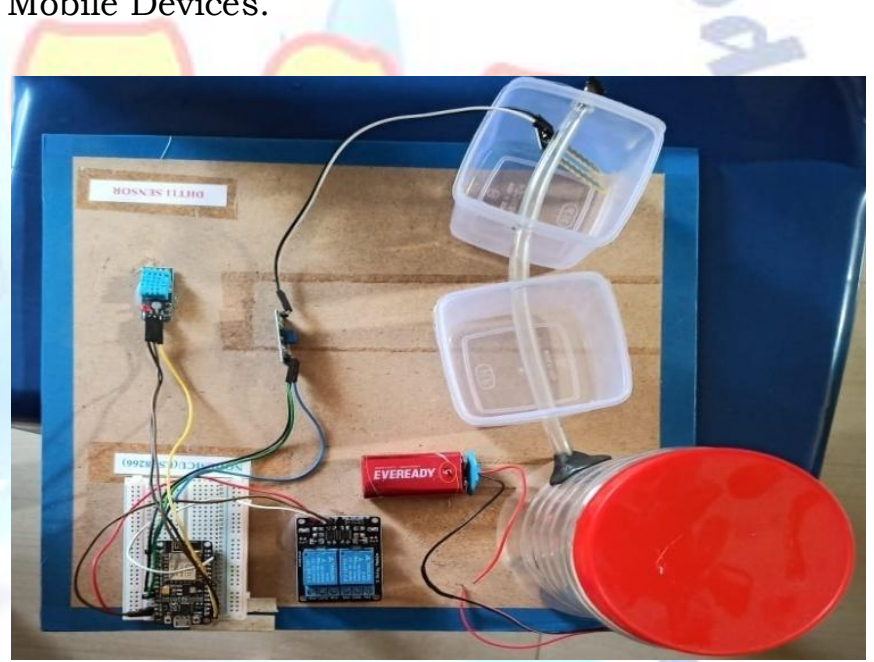

Fig 6.2: Dynamic Planting \& Farming

The above diagram depicts the Dynamic planting and farming that consists of node MCU, DHT sensor, Soil moisture sensor, Relay pin, Battery and motor. Based on the soil moisture the motor turned on and off for smart planting and farming and also user can control or view the device status using web app or Mobile Devices. 


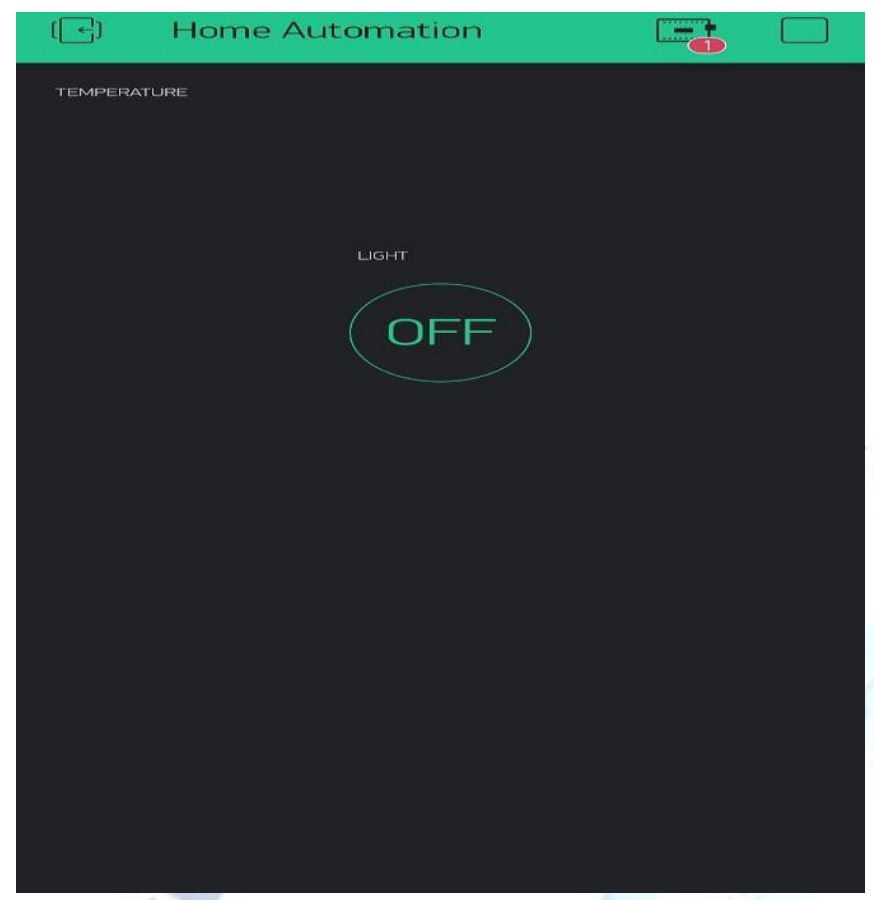

Fig 6.3: Light Control Interface

The above diagram depicts the Light Control interface that consists of icon light pin. Based on the user input the object can control or view for the result.

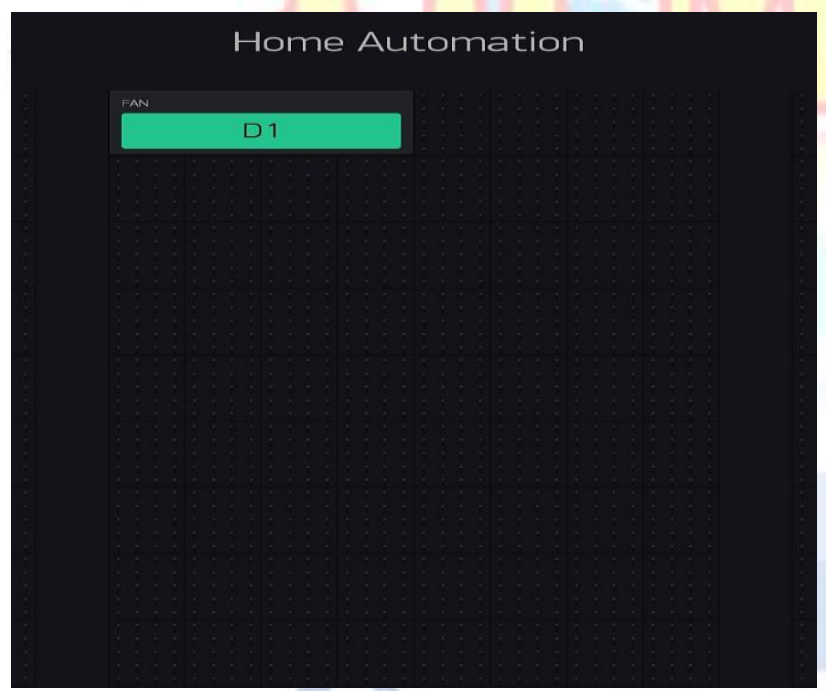

Fig 6.4: Fan Control Interface

The above diagram depicts the Fan Control interface that consists of icon fan pin. Based on the user input the object can control or view for the result.

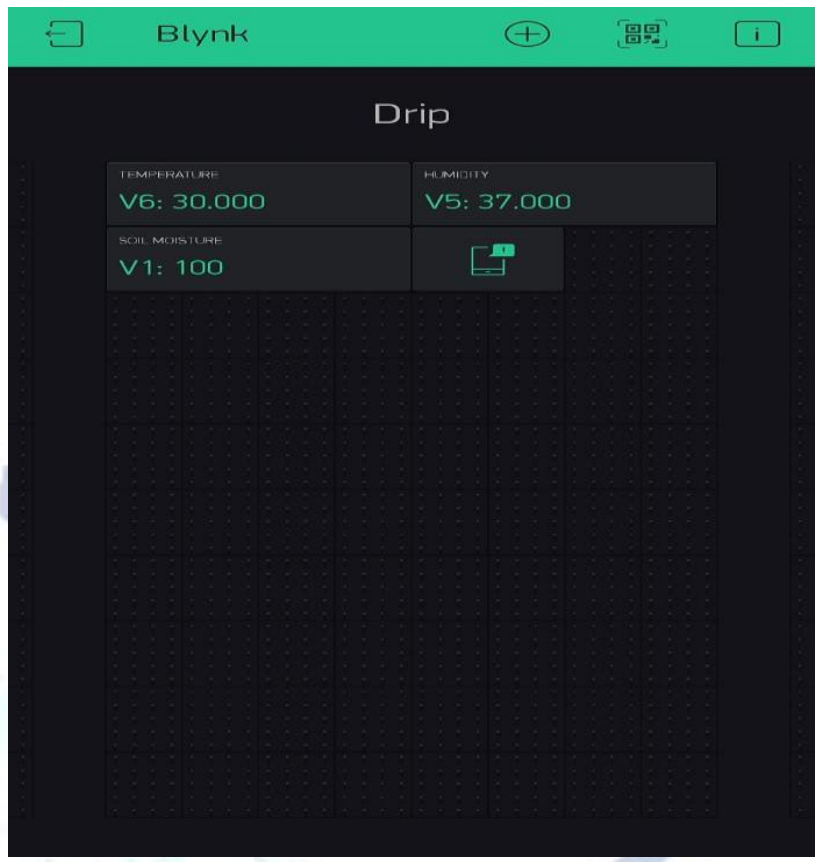

Fig 6.5: Smart Farming interface

The above diagram depicts the smart farming interface that consists of icon as temperature, humidity, soil moisture pin. Based on the user input the object can control or view for the result.

\section{Conclusion}

In this paper, a home automation system and smart plantation or farming system was designed and implemented using Node MCU microcontroller enables with $\mathrm{Wi}-\mathrm{Fi}$ as the method of monitoring and controlling the home appliances i,e user remotely access the system from anywhere around the world. The system is capable of automating the operation of the appliances by analyzing the regular usage patter of the appliances by the user. This not just saves a lot of human effort, but also helps in conserving energy. Also, it can help the differently abled and the elderly in performing basic tasks at home such as switching on/off the light, fan, and so on without having to depend on others.

\section{FUTURE WORK}

IoT involves enhancing network to proficiently collect and analyze the data from various sensors and actuators then send the data to the mobile phone of a personal computers over a wireless connection, building IoT has progressed essentially in the last couple of years since it has created a new era in the world of information's and communication technologies . Security is an important issue now days, as the possibilities of intrusion are increasing day by day. Safety from intrusion, theft, fire and leakage gas are most 
important requirements of home security system for the peoples.

\section{ACKNOWLEDGMent}

I thank my Mentor Prof. Chethan Raj C for analyzing the data and for advising on all aspects related to this paper, along with other concepts too. Later for validating the experimental result and reviewed the paper.

\section{REFERENCES}

[1] "Home Automation and Security System with Node MCU using Internet of Things", K. Lova Raju, V. Chandrani, SK. Shahina Begum, M. Pravallika Devi, IEEE 2019.

[2] "Smart-Home Automation using IoT-based Sensing and Monitoring Platform", Majid Al-Kuwari, Abdulrhman Ramadan, Yousef Ismael, Laith Al-Sughair, Adel Gastli, Mohieddine Benammar ,IEEE 2019.

[3] "Smart Energy Efficient Home Automation System Using IoT", Satyendra K. Vishwakarma, Prashant Upadhyaya, Babita Kumari, Arun Kumar Mishra, IEEE 2019.

[4] "A Review on Home Automation using Voice Via Bluetooth Through Raspberry PI3”, Jayant Dorve, Manish K. Samarth, Swapnil R. Jais, Md. Danish S. Sheikh, Pawan Kumar, Hanuman Korde, IJRESM 2019.

[5] "Smart Irrigation System Using Machine Learning and IOT", Revanth Kondaveti, Akash Reddy, Supreet Palabtla , IEEE 2019.

[6] "Advanced Irrigation System using Arduino and Raspberry Pi as Centralized Server",N. Kishore, K. Ashok kumar, IRJET 2019.

[7] "Smart Gardening Automation using IoT With BLYNK App" , Mitul Sheth, Pinal Rupani ,IEEE 2019.

[8] "Home Automation System Based on IOT using Cellular Devices", Ravi Wankhade, Shashank Karhade, Pratik Mohite, Kanchan Dhole, Akash Ganvir, Bharti Khedkar, Sharayu Sangekar, IJSRST 2019.

[9] "IoT Based Smart Home Garden Watering System Using Raspberry Pi 3", Sandhya.B.R, Pallavi.M, Chandrashekar.M, IJIRSET 2018.

[10] "Arduino based automatic water planting system using soil moisture sensor", Hriday Chawla, Praveen Kumar, ICAESMT 2019.

[11] "Cost Effective Autonomous Plant Watering Robot", Mahendra Vucha, K Jyothi, Kiran Kumari, R Karthik, IJRTE 2019.

[12] "WaterIrrigationSystemusingArduino",Mr.Muthamilselvan, Narendra Kumar Meel, Chetan Sharma , Arshad Ali, IRJET 2018 